\title{
Towards a more rapid diagnosis of rapidly progressive glomerulonephritis
}

\author{
Michael J D Cassidy, Gillian Gaskin, John Savill, Charles D Pusey, Andrew J Rees
}

\begin{abstract}
An attempt was made to provide simple practical guidelines to alert general practitioners to the diagnosis of rapidly progressive glomerulonephritis and lead to early referral to hospital. The duration of illness before referral to this hospital and its effect on outcome in patients with crescentic nephritis were assessed retrospectively from the case notes of 24 patients referred over two years. Four patients had Goodpasture's syndrome, 11 Wegener's granulomatosis, seven microscopic polyarteritis, and two idiopathic progressive glomerulonephritis. The duration of symptoms before referral to the local hospital was similar in the four groups of patients and varied from one week to 28 months (mean 10 months). The duration of stay in the local hospital was two, nine, 11, and 180 days in the patients with Goodpasture's syndrome and a mean of four days (range one to eight) in those with Wegener's granulomatosis and 10 days (one to 18 days) in those with microscopic polyarteritis. In the local hospital the diagnosis was based on the results of renal biopsy and detection of antibodies to glomerular basement membrane in two patients with Goodpasture's syndrome and on the results of renal biopsy in seven of the other patients aided by the detection of antibodies to the cytoplasm of neutrophils (ANCA) in 10 . Three of the 24 patients died and four required maintenance haemodialysis.
\end{abstract}

Patients who present to their general practitioners with persistent non-specific symptoms should have a urine dipstick test and then blood tests and emergency referral to hospital if necessary. Hospital physicians should be aware of the speed and accuracy with which current assays can confirm a diagnosis of rapidly progressive glomerulonephritis.

\section{Introduction}

Rapidly progressive glomerulonephritis, the clinical counterpart of focal necrotising glomerulonephritis with epithelial crescents, is an aggressive condition that can occur in a wide variety of clinical diseases but is most often associated with either systemic vasculitis or circulating antibodies to glomerular basement membrane (Goodpasture's syndrome). ${ }^{1}$ Recently antibodies to the cytoplasm of neutrophils (ANCA) have been detected in the serum of a high proportion of patients with systemic vasculitis. There are at least two types: antibodies to components of cytoplasm (c-ANCA), which occur in classical Wegener's granulomatosis, and antibodies to components of the perinuclear region of cytoplasm (p-ANCA), which are found in patients with microscopic polyarteritis and idiopathic rapidly progressive glomerulonephritis. ${ }^{2}$ Serological tests are extremely useful when making a diagnosis, but ultimately this must be based on the results of renal biopsy.
If untreated rapidly progressive glomerulonephritis results in dialysis or death in over $90 \%$ of patients. Specific treatment with corticosteroids, cytotoxic agents, and, in severe cases, plasma exchange has considerably improved the outlook. Even so, recovery of renal function in patients who have progressed to needing dialysis rarely occurs in those with Goodpasture's syndrome despite aggressive treatment; this is not true in those with Wegener's granulomatosis or microscopic polyarteritis, in whom aggressive treatment is often rewarding. ${ }^{3}$

We retrospectively analysed data from the case notes of such patients with crescentic nephritis referred to our unit over two years to assess the duration of illness before referral and the effect on outcome. We aimed to provide simple practical guidelines to alert general practitioners to the potential diagnosis and lead to early hospital referral.

\section{Patients and methods}

From January 1988 to December 1989, 24 patients (nine men, 15 women) with rapidly progressive glomerulonephritis were referred to this hospital from other hospitals. The mean age of the group was 56 (range 22 to 76). Four patients had Goodpasture's syndrome (positive results of tests for antibodies to glomerular basement membrane and linear deposition of IgG on the glomerular basement membrane in a renal biopsy specimen); 11 had Wegener's granulomatosis; seven had microscopic polyarteritis as previously described ${ }^{45}$; and two were classified as having idiopathic rapidly progressive glomerulonephritis. All 20 patients who did not have Goodpasture's syndrome had antibodies to the cytoplasm of neutrophils detected by indirect immunofluorescence. The duration of symptoms consistent with their underlying disease before referral to their local hospital was recorded, as was the serum creatinine concentration at presentation. The duration of attendance or admission at the local hospital before referral to our unit was noted. These data were analysed with special reference to outcome.

\section{Results}

The duration of symptoms before referral to the local hospital varied from one week to 28 months (mean 10 months). The mean duration was similar in all three categories of disease: 10 months in the patients with Goodpasture's syndrome, 11 months in those with Wegener's granulomatosis, and eight months in those with microscopic polyarteritis and idiopathic rapidly progressive glomerulonephritis.

All four patients with Goodpasture's syndrome had serum creatinine concentrations of less than $700 \mu \mathrm{mol} / \mathrm{l}$ at presentation to their local hospital, but by the time of referral to our unit all required dialysis. Their duration of stay at the local hospital was two, nine, 11, and 180 
days. In two patients the diagnosis was made on the basis of a positive result of an assay for antibodies to glomerular basement membrane and findings on renal biopsy before transfer. Despite plasma exchange and immunosuppressive treatment ${ }^{3}$ one patient died and two required maintenance dialysis. One patient recovered renal function.

The average duration of stay in hospital before referral was four days (range one to eight days) for the patients with Wegener's granulomatosis and 10 days (range one to 18 days) for those with microscopic polyarteritis. The diagnosis was based on the results of renal biopsy at the referring hospital in seven of the 20 patients, and immunosuppressive treatment was started in 12. The diagnosis was made or supported by detection of antibodies to the cytoplasm of neutrophils in 10 of the 20 serum samples analysed before transfer. Three patients were already receiving dialysis at the time of transfer and dialysis became necessary in a
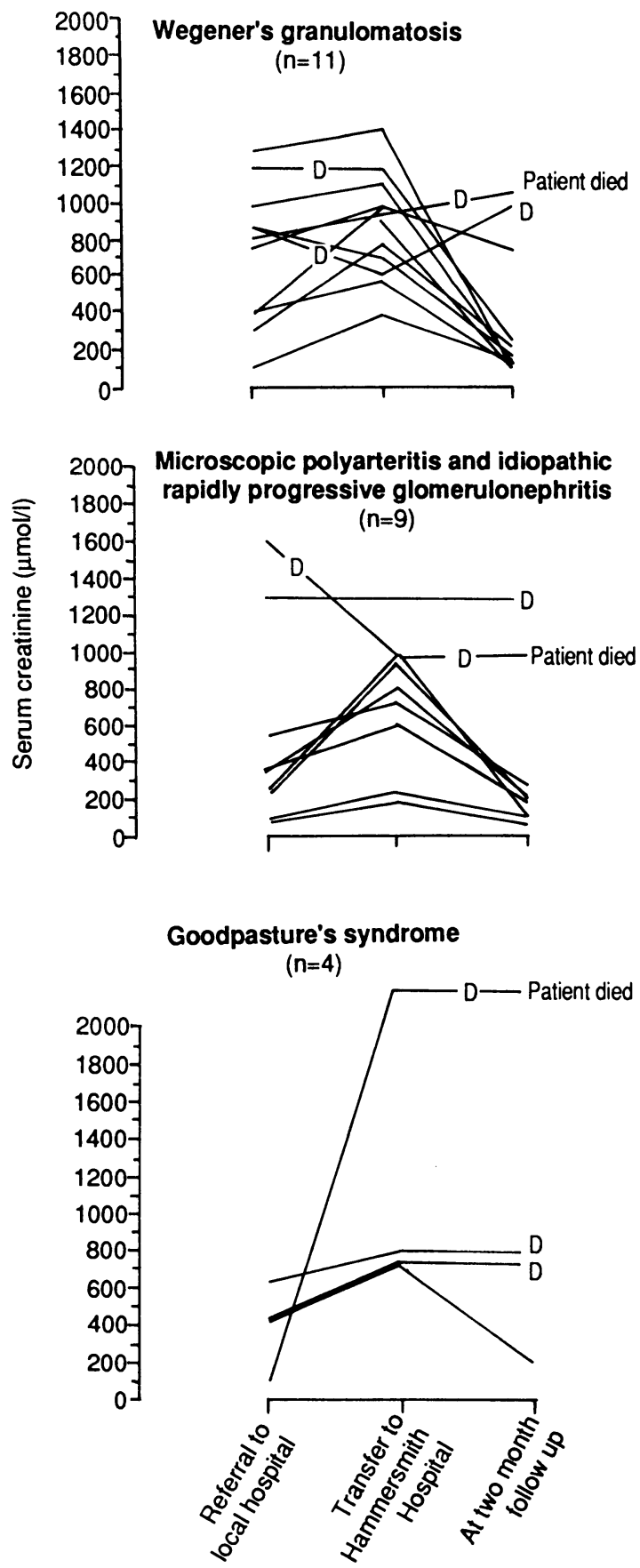

FIG 1-Serum creatinine concentration in patients with Wegener's granulomatosis, microscopic polyarteritis and idiopathic rapidly progressive glomerulonephritis, and Goodpasture's syndrome at three stages after presentation.

$D=$ Dialysis

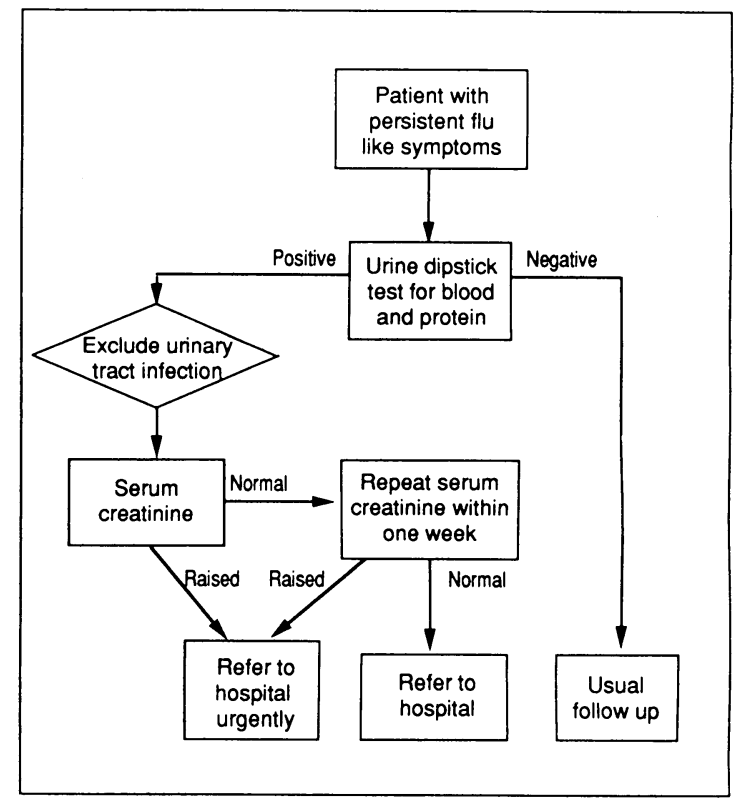

FIG 2-Procedures to lessen risk of missing diagnosis of rapidly progressive glomerulonephritis in general practice

further four. Two patients died and two required maintenance dialysis. At two month follow up renal function had improved in the remaining patients (fig 1).

\section{Discussion}

In most patients initial symptoms were non-specific, such as malaise and joint pains; as expected, ear, nose, and throat symptoms were common in the patients with Wegener's granulomatosis. Initially, it would be difficult to distinguish these symptoms from those of a common viral infection. A multisystem disease affecting the kidneys was not considered for some time in many cases: symptoms persisted for more than four weeks in over $80 \%$ of the patients.

Urine analysis is crucial to early diagnosis. Blood and protein are invariably present in the urine and can be readily detected by dipstick testing. Microscopical examination of a fresh urine sample should be mandatory in hospital in such cases as red cells and casts further suggest active glomerulonephritis. We realise that for a general practitioner microscopical examination of urine, though desirable, requires additional equipment and time and that time is in short supply; at this stage dipstick testing is enough to alert suspicion. Renal function can then be checked: the serum creatinine concentration, a more reliable marker of intrinsic renal disease than urea concentration, is usually reported, together with other biochemical variables measured with an autoanalyser. Other markers of active vasculitis are a low serum albumin concentration and an increased alkaline phosphatase activity. Once a clinically suspected diagnosis of rapidly progressive glomerulonephritis is confirmed the patient should be treated as a medical emergency. Immediate referral to hospital is necessary, where a full clinical and serological investigation including renal biopsy will confirm the diagnosis.

Serological investigations such as specific solid phase immunoassays for circulating antibodies to glomerular basement membrane have been available for several years and permit rapid diagnosis of Goodpasture's syndrome. More recently, antibodies to cytoplasmic constituents of neutrophils and monocytes have been described. These have been reported to have sensitivities and specificities of over $90 \%$ in active Wegener's granulomatosis and microscopic polyarteritis. ${ }^{2}$ In fact, the presence of these antibodies was the basis for 
diagnosis before referral in half of the patients in this study. Indirect immunofluorescence or solid phase immunoassays to detect antibodies to the cytoplasm of neutrophils are becoming more widely available and have greatly helped in diagnosis.

We suggest that patients who present to their general practitioners with persistent non-specific symptoms should have a urine dipstick test followed by blood tests and urgent referral to hospital if necessary; figure 2 shows an algorithm to illustrate this. Hospital doctors should be aware of the speed and accuracy with which current assays can confirm a diagnosis of rapidly progressive glomerulonephritis. This approach should reduce the morbidity and mortality associated with this group of diseases.

1 Pusey CD, Lockwood CM. Autoimmunity in rapidly progressive glomerulonephritis. Kidney Int 1989;35:929-37.

2 Van der Woude FJ, Daha MR, Van Es LA. The current status of neutrophil cytoplasmic antibodies. Clin Exp Immunol 1989;78:143-8.

3 Hind CKR, Paraskevakou H, Lockwood CM, et al. Prognosis after immunosuppression of patients with crescentic glomerulonephritis requiring dialysis. suppression of patient
Lancet 1983;ii:263-6.

4 Pinching AJ, Lockwood CM, Pussell BA, et al. Wegener's granulomatosis: observations on 18 patients with severe renal disease. Q $\mathcal{F}$ Med 1983;208: $435-60$

5 Savage COS, Winearls CG, Evans DJ, Rees AJ, Lockwood CM. Microscopic polyarteritis: presentation, pathology and prognosis. $Q \mathcal{F}$ Med 1985;56: $467-84$

(Accepted 23 May 1990)

\title{
Lesson of the Week
}

\section{Wilson's disease in adults with cirrhosis but no neurological abnormalities}

\author{
D M Danks, G Metz, R Sewell, E J Prewett
}

Adults with Wilson's disease may present with liver disease without neurological symptoms
Murdoch Institute, Royal Children's Hospital,

Parkville 3052, Melbourne,

Australia

D M Danks, FRACP, director

Alfred Hospital, Melbourne, Australia

G Metz, FRACP, gastroenterologist

Austin Hospital, Melbourne, Australia R Sewell, FRACP, gastroenterologist

St Vincent's Hospital, Melbourne, Australia E J Prewett, MB, medical registrar

Correspondence to: Dr Danks.
The classic description of Wilson's disease is a progressive dystonic neurological disorder in young adults who have a chronic liver disease determined by examination, investigation, or at necropsy. A progressive accumulation of copper in the liver and spillover to the brain is well established. The cause of the hepatic copper retention remains unknown, although defective incorporation of copper into caeruloplasmin and decreased biliary excretion of copper have been suggested. ${ }^{12}$ It is well accepted that patients with Wilson's disease often present with liver disease alone in childhood and early adult life, and it is generally agreed that Wilson's disease is the most common definable cause of chronic liver disease in later childhood. ${ }^{13}$ The fallibility of the standard diagnostic tests for Wilson's disease in this age group is also well recognised: Kayser-Fleischer rings may be absent and serum copper and caeruloplasmin concentrations may be normal or near normal..$^{1-3}$ The copper content of the liver and profile of clearance of copper-64 from the blood $^{4}$ must be estimated in these cases.

Wilson's disease is not generally considered in patients aged over 30 presenting with liver disease without neurological signs, and the fallibility of standarc tests in such patients is not well recognised. Indeed, most reports of large series of patients with Wilson's disease include no such case. Scheinberg and Sternlieb mention two patients aged 30 to 35,2 and another study briefly mentions four patients with onset at $37,37,47$, and 55 years of age but gives no clinical details or results of tests. ${ }^{5}$ The man aged 55 was probably the same one described in another report ${ }^{6}:$ he had Kayser-Fleischer rings, low serum caeruloplasmin and copper concentrations, and an abnormal rate of copper clearance.

We report on four adults with Wilson's disease aged 43-58 who presented within 12 months with chronic or acute on chronic liver disease without neurological symptoms in whom assay of hepatic copper content or clearance of ${ }^{6+} \mathrm{Cu}$ from the blood, or both, were required to diagnose their condition.

\section{Case reports}

Case 1-A man aged 43 presented with general malaise, loss of energy, and mild jaundice. He had a history of pulmonary sarcoidosis, which had been diagnosed when he was 34 and treated with corticosteroids; vitiligo for 10 years; and steatorrhoea due to pancreatic insufficiency, which had been diagnosed when he was 40 and treated with pancreatic enzyme replacement. None of the known hepatitis viruses could be identified, autoantibody tests yielded negative results, and his alcohol intake was minimal. Kayser-Fleischer rings were not visible with a slit lamp, and his serum copper concentration was normal $(24 \mu \mathrm{mol} / \mathrm{l})$. A liver biopsy showed cirrhosis with no features of cholestasis or primary biliary cirrhosis, but the copper content had not been measured. His rate of clearance of ${ }^{\circ} \mathrm{Cu}$ was measured by injecting the isotope intravenously and taking serum samples at 0,5 , and 30 minutes and $1,2,4,24$, and 48 hours. The results were typical of Wilson's disease (figure). After six

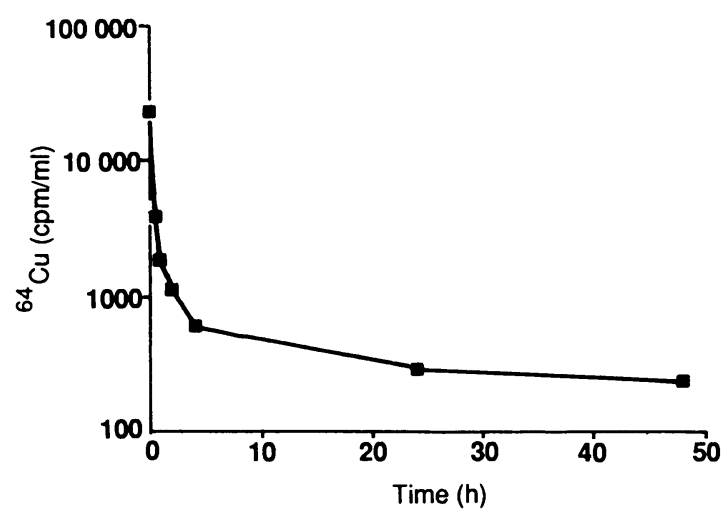

Clearance of intravenously injected ${ }^{m} \mathrm{Cu}$ in patient with Wilson's disease

months of treatment with penicillamine $(500 \mathrm{mg}$ twice daily) and zinc sulphate $(220 \mathrm{mg}(50 \mathrm{mg}$ zinc) three times daily) his liver function improved. Wilson's disease was excluded in his younger sister by testing her clearance of ${ }^{64} \mathrm{Cu}$.

Case 2-A 48 year old man was diagnosed as suffering from cirrhosis after hepatomegaly was noted as an incidental finding. He had no relevant symptoms or history. He had never drunk alcohol, none of the known hepatitis viruses could be identified, and no autoantibodies were detected. He had no Kayser-Fleischer rings, and his serum copper and caeruloplasmin concentrations were normal $(22 \mu \mathrm{mol} / \mathrm{l}$ 